\title{
EDITORIAL
}

\section{The cultural revolution}

\author{
Despite the revolutionary advances made through the application of omic approaches, the \\ importance of studying organisms in pure culture should not be forgotten.
}

Metagenomics is often said, not least by us, to have revolutionised microbiology, and indeed over the past decade metagenomic analyses have revealed the astounding diversity of microbial life that can be found in a range of environments, from oceans and soils to the human supraorganism. Moreover, with technical developments in next-generation sequencing increasing the speed and decreasing the cost of sequencing at an astonishing rate, whole-genome sequencing studies are now en vogue, and there are some excellent recent examples of using wholegenome sequencing to study the molecular epidemiology of infectious disease. New technologies can be seductive, however, and it is perhaps a little too easy to forget that a firm grounding in basic principles, such as bacterial physiology and metabolism, is required to exploit these techniques most effectively.

One example of this neglect of basic principles is the fact that bacterial culturing has somewhat fallen by the wayside in research laboratories, having been superseded first by $16 \mathrm{~S}$ rRNA analysis and then by metagenomics. Thankfully, there are exceptions to this trend, and a number of researchers still undertake culture-dependent analyses hand in hand with culture-independent, genomicsbased studies. At Oregon State University, USA, Stephen Giovannoni has been interested in high-throughput culture methods for many years and has developed a highthroughput microbial cultivation facility. One of the major research interests of the Giovannoni laboratory is the SAR11 clade of alphaproteobacteria (marine heterotrophs that are some of the most abundant organisms on the planet and have an important role in nutrient cycling). A member of the SAR11 clade, 'Candidatus Pelagibacter ubique str. HTCC1062', was first obtained in pure culture a decade ago $^{1}$ in an undefined natural seawater medium. This development greatly facilitated research on this species, but the use of an undefined medium did lead to problems, such as a limited ability to draw comparisons between experiments conducted using different batches of medium.

In a recent article, Giovannoni and colleagues report the development of a defined artificial seawater medium for ' $C a$. Pelagibacter ubique', containing inorganic salts, vitamins, methionine, glycine and pyruvate ${ }^{2}$. The requirements for methionine (as a source of reduced sulphur) and for glycine were known, but the requirement for pyruvate as the last essential macronutrient was a new finding. The process involved in developing the defined medium revealed several insights into the unusual metabolism of 'Ca. Pelagibacter ubique', which will lead to the refinement of the current metabolic models for this organism.

Not all organisms are as fastidious as ' $\mathrm{Ca}$. Pelagibacter ubique'. In the environment, microbial communities are dominated by low-abundance taxa (the so-called rare biosphere). It is often assumed that these microorganisms are difficult to culture. However, Shade, Handelsman and colleagues ${ }^{3}$ recently used both culture-based and cultureindependent assays to assess the contribution of lowabundance organisms to soil community structure. They found that using standard cultivation conditions, the culture-based approach captured more members of the rare biosphere than pyrosequencing, demonstrating that the nutritional requirements of rare-biosphere members are not necessarily as eclectic as one might imagine.

Of course, the advantages of culturing don't apply only to environmental microbiology. In medical microbiology, there have been some notable successes in obtaining intracellular pathogens in axenic (host cell-free) culture. Raoult and colleagues developed an axenic medium for Tropheryma whipplei, the causative agent of Whipple's disease, through metabolic reconstruction from the genome sequence 4 . A systematic approach was also used to develop an axenic culture system for Coxiella burnetii, the causative agent of Q fever ${ }^{5}$. Most recently, an axenic culture medium that will support the growth of the two developmental forms of Chlamydia trachomatis (infectious elementary bodies (EBs) and vegetative reticulate bodies (RBs)) was developed ${ }^{6}$. This allowed the energy requirements of both forms to be compared, revealing that EBs require glucose-6-phosphate and RBs require ATP.

The insights that have arisen from culture-independent analyses have been phenomenal, but to truly be able to verify these insights, the organisms must be grown in the laboratory. Maybe it's time for more of us to join the cultural revolution?

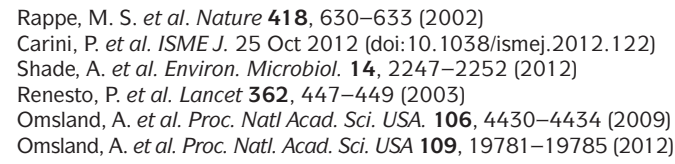

